\title{
OPEN Endometrial microbiota is more diverse in people with endometriosis than symptomatic controls
}

\author{
Jocelyn M. Wessels ${ }^{1}$, Miguel A. Domínguez ${ }^{2}$, Nicholas A. Leyland ${ }^{1}$, Sanjay K. Agarwal ${ }^{3}$ \& \\ Warren G. Foster ${ }^{1 \bowtie}$
}

Endometriosis is a chronic, estrogen-dependent gynecological condition affecting approximately $10 \%$ of reproductive age women. The most widely accepted theory of its etiology includes retrograde menstruation. Recent reports suggest the uterus is not sterile. Thus, the refluxed menstrual effluent may carry bacteria, and contribute to inflammation, the establishment and growth of endometriotic lesions. Here, we compared and contrasted uterine bacteria (endometrial microbiota) in people with surgically confirmed presence $(N=12)$ or absence of endometriosis $(N=9)$ using next-generation $16 S$ rRNA gene sequencing. We obtained an average of $>9000$ sequence reads per endometrial biopsy, and found the endometrial microbiota of people with endometriosis was more diverse (greater Shannon Diversity Index and proportion of 'Other' taxa) than symptomatic controls (with pelvic pain, surgically confirmed absence of endometriosis; diagnosed with other benign gynecological conditions). The relative abundance of bacterial taxa enriched in the endometrial microbiota of people with endometriosis belonged to the Actinobacteria phylum (Gram-positive), Oxalobacteraceae (Gramnegative) and Streptococcaceae (Gram-positive) families, and Tepidimonas (Gram-negative) genus, while those enriched in the symptomatic controls belonged to the Burkholderiaceae (Gram-negative) family, and Ralstonia (Gram-negative) genus. Taken together, results suggest the endometrial microbiota is perturbed in people with endometriosis.

Endometriosis is a complex disease that affects approximately $10 \%$ of women of reproductive age, and often associated with the main clinical features of pelvic pain (mechanisms involved in pain and endometriosis reviewed $i^{1}$ ) and infertility. It is caused by development of endometrial-like glands and stroma outside the uterus, and though its exact pathogenesis remains unclear, it appears to involve a combination of contributing factors such as retrograde menstruation into the peritoneal cavity ${ }^{2}$, and an altered immune response $\mathrm{e}^{3,4}$. Other theories on the origin of endometriotic lesions include the embryonic rest theory (prenatal endometrial precursor cells differentiate and become established in the pelvic region $)^{5-7}$, coelomic metaplasia (transformation of the peritoneal mesothelium), lymphovascular metastasis (transportation of endometrial cells via lymphatics or blood), or endometrial stem/progenitor cells (reviewed $\mathrm{in}^{8-10}$ ). However, other factors (anatomical, genetic, environmental, lifestyle, menstrual cycle dynamics, aberrant immune responses, etc. $)^{10-14}$ are likely involved as $90 \%$ of women experience retrograde menstruation ${ }^{15}$, but only about $10 \%$ develop endometriosis ${ }^{13}$. Recent studies have also suggested serum metabolites are altered in endometriosis ${ }^{16}$, that genetic/epigenetic changes caused by retrograde menstruation into the peritoneal cavity contribute to lesion development (reviewed $\mathrm{in}^{17}$ ), and that genetic predisposition may differ in relation to ethnicity ${ }^{18}$.

Even though the human body is home to more than ten times more bacteria than nucleated human cells (ratio is 1:1 if compared with all cells in the body, due to many non-nucleated red blood cells) ${ }^{19,20}$, for years the uterus was thought to be sterile and not contain bacteria. This was likely because bacterial culture was the main technique employed to identify bacteria. However, many bacterial species are difficult, if not impossible, to grow in vitro due to nutritional or other environmental requirements. Nevertheless, a few bacterial culture studies in

${ }^{1}$ Department of Obstetrics \& Gynaecology, McMaster University, Hamilton, ON L8S 4K1, Canada. ${ }^{2}$ Facultad de Medicina Veterinaria y Zootecnia, Universidad Autónoma de Tamaulipas, 87000 Cd. Victoria, TAMPS, Mexico. ${ }^{3}$ Department of Reproductive Medicine, University of California San Diego, La Jolla, CA 92037, USA. ${ }^{\circledR}$ email: fosterw@mcmaster.ca 
females undergoing in vitro fertilization (IVF) hinted at the existence of an endometrial (uterine) microbiota when the presence of bacteria on embryo transfer catheters was negatively associated with IVF success ${ }^{21-23}$. With the advent of advanced molecular biology techniques like 16S rRNA gene sequencing, the identification of microorganisms in the microbiotas collected from different sites of the body has become fairly routine. Indeed, even the long-held belief that the uterine environment was sterile has been challenged with next generation sequencing reports of microbial signatures in the uterus ${ }^{24-33}$. However, because these studies mainly included fertility patients or those with gynecological disease, there is some uncertainty surrounding the endometrial microbiota of "healthy" women. Some of the presently contested points include (1) the source of endometrial microbiota seeding, (2) the existence of a stable, resident endometrial microbiota in the "healthy" state, (3) the bacterial species included in a normal/"healthy" endometrial microbiota (if it exists), and (4) whether uterine bacteria are transient and only associated with pathologies (reviewed in $^{34}$ ). Nevertheless, it appears as though bacteria participate in uterine-related diseases like endometriosis ${ }^{26,31,33}$, endometrial cancer ${ }^{35,36}$, and uterine fibroids ${ }^{30}$. In fact, reports of bacterial endotoxin in the pelvic cavity and menstrual blood of people with endometriosis ${ }^{37}$ have led to a bacterial contamination hypothesis suggesting endotoxin/bacteria in the menstrual effluent contributes to pelvic inflammation, growth, and progression of endometriotic lesions (reviewed $\mathrm{in}^{38,39}$ ). Furthermore, several studies suggest the microbiotas (gut, vaginal, cervical, uterine/endometrial) of patients with endometriosis differ females without this condition ( $\left(\right.$ reviewed $\mathrm{in}^{40}$ ). Therefore, the objective of the present study was to compare the endometrial microbiota recovered from endometrial biopsies of patients with surgically confirmed presence or absence of endometriosis (cases versus symptomatic controls-patients with pain but without endometriosis). We chose to include symptomatic controls, rather than asymptomatic controls, because of the aforementioned uncertainty surrounding the endometrial microbiota of "healthy" women, and because one of our aims was to examine differentially expressed taxa that might be unique to patients with endometriosis as compared to other gynecological conditions.

\section{Materials and methods}

Study participants. The study was approved by the Research Ethics Board, McMaster University (Institutional Review Board no. 06-064, 14-066-T), and all participants provided written informed consent and basic demographic/gynecological history prior to participation. All methods were performed under the approved study protocol, in accordance with the relevant guidelines and regulations. In this prospective, cross-sectional study 24 patients attending McMaster University Medical Centre were selected for the present study from a larger study on endometriosis (2011-2017). This study size was determined based on our previous work in the vaginal microbiota where differences in the microbiota could be observed with a group size of ten ${ }^{41}$. A sample size calculation was also performed. We anticipate a difference between groups of approximately 1.2 on the Shannon Diversity Index, and a standard deviation of 0.8 , based on our previous study ${ }^{41}$. A sample size calculation using a two-tailed t-test to achieve a power of $80 \%$ and alpha of $5 \%$, with a $\mathrm{SD}=0.8$ and expected difference of 1.2 between groups, indicates that 9 women per study group would be required for the present study. Patients at our tertiary care centre were undergoing gynecological laparoscopy for pelvic pain thought to be due to endometriosis. During surgery patients were categorized as a case or symptomatic control (people with pain but no surgical or pathological evidence of endometriosis) by the gynecological surgeon (NAL), and diagnoses were confirmed by histopathology. Of the 24 patients recruited, 14 were diagnosed with endometriosis (Cases; Stage 1: 0, Stage 2: 1, Stage 3: 1, Stage 4: 12) using the revised American Fertility Score (rAFS) ${ }^{42}$, while 10 were diagnosed with other benign gynecological conditions (Symptomatic Controls). Exclusion criteria were people unable to provide consent, aged under 18, currently pregnant, or who had used hormone therapies (oral contraceptives, GnRH agonist/antagonist, progestins, etc.) in the 3 months preceding study enrollment.

Endometrial biopsy collection. Immediately before surgery the vagina was swabbed with chlorhexidine in preparation for the gynecological laparoscopy. A sterilized vaginal speculum was inserted, and then a double sheathed, sterile pipelle endometrial suction curette (Cooper Surgical, Trumbull, CT, USA) was passed through the cervix to collect an endometrial biopsy, taking care to avoid contact with the vaginal wall and cervix. Biopsies were deposited in sterile $15 \mathrm{~mL}$ Falcon conical tubes (polystyrene) (Fisher Scientific, Ottawa, ON, Canada) and transported to the laboratory on ice, where they were processed within $30 \mathrm{~min}$. One portion of the endometrial biopsy was fixed in $10 \%$ buffered formalin (Staplex Scientific, Etobicoke, ON, Canada) and processed for routine histology. Slides were cut for each biopsy in $5 \mu \mathrm{m}$ sections, and stained with hematoxylin and eosin to confirm menstrual cycle phase using the Noyes criteria ${ }^{43}$. A second portion of the endometrial biopsy was placed in RNAlater (Sigma-Aldrich Canada, Oakville, ON, Canada), kept at $4{ }^{\circ} \mathrm{C}$ overnight, and then stored at $-80{ }^{\circ} \mathrm{C}$ until processed for nucleic acid extraction.

Bacterial V3 region of 16S rRNA gene sequencing. Biopsies were thawed, weighed (10-50 mg) and homogenized in $700 \mu \mathrm{L}$ Qiazol lysis buffer (Qiagen, Hilden, Germany) using a Pro200 tissue homogeniser (PRO Scientific, Oxford, CT, USA). Total nucleic acid extraction was performed using the RNeasy Mini Kit (Qiagen, Hilden, Germany), omitting the DNase treatment, and following the manufacturer's protocol. The quantity and purity of nucleic acids was assessed using the Nanodrop 2000 (Thermo Fisher Scientific, Burlington, ON, Canada). A final volume of $80 \mu \mathrm{L}$ was frozen and stored at $-80^{\circ} \mathrm{C}$ until required for sequencing. Samples were numerically coded and researchers were blinded to experimental groups until data analysis.

To retain bacterial DNA and eliminate RNA that might inhibit the PCR reaction, RNase A (Qiagen Hilden, Germany) was added to the first PCR mastermix. The hypervariable V3 region of the $16 \mathrm{~S}$ rRNA gene was amplified using a two-stage (nested) PCR approach. Initially the 8f. (AGAGTTTGATCCTGGCTCAG) to 1492r (CAC GGATCCTACGGGTACCTTGTTACGACTT) region of the $16 \mathrm{~S}$ rRNA gene was amplified in triplicate using 
100-200 ng of DNA template with $2 \mathrm{U}$ of Taq, $1 \times$ buffer, $1.5 \mathrm{mM} \mathrm{MgCl}_{2}, 0.4 \mathrm{mg} / \mathrm{mL} \mathrm{BSA}, 0.2 \mathrm{mM} \mathrm{dNTPs}, 50 \mu \mathrm{g} /$ $\mathrm{mL}$ RNaseA, and $10 \mathrm{pmols}$ of each primer. The initial PCR reaction was carried out at $94^{\circ} \mathrm{C}$ for $5 \mathrm{~min}, 15$ cycles of $94^{\circ} \mathrm{C}$ for $30 \mathrm{~s}, 56^{\circ} \mathrm{C}$ for $30 \mathrm{~s}$ and $72{ }^{\circ} \mathrm{C}$ for $90 \mathrm{~s}$, with a final extension of $72{ }^{\circ} \mathrm{C}$ for $10 \mathrm{~min}$. The triplicate reaction was then combined and used as the template in the second stage of the nested PCR. In the second PCR, $3 \mu \mathrm{L}$ of the first PCR reaction product was used as the template and was combined with $2 \mathrm{U}$ of Taq, $1 \times$ buffer, $1.5 \mathrm{mM}$ $\mathrm{MgCl}_{2}, 0.4 \mathrm{mg} / \mathrm{mL}$ BSA, $0.2 \mathrm{mM}$ dNTPs, and 5pmols each of Illumina adapted primers 341F (CCTACGGGA GGCAGCAG) and 518R (ATTACCGCGGCTGCTGG) (primers + Illumina adapters/barcode/priming region as described in supplemental materials of Bartram et al., 2011: $80 \mathrm{bp})^{44-46}$. The PCR reaction was carried out at $94{ }^{\circ} \mathrm{C}$ for $5 \mathrm{~min}, 35 \mathrm{cycles}$ of $94^{\circ} \mathrm{C}$ for $30 \mathrm{~s}, 50^{\circ} \mathrm{C}$ for $30 \mathrm{~s}$, and $72{ }^{\circ} \mathrm{C}$ for $30 \mathrm{~s}$, with a final extension of $72{ }^{\circ} \mathrm{C}$ for $10 \mathrm{~min}$. Each PCR run contained no template negative controls (sterile water), which did not yield PCR products (no $300 \mathrm{bp}$ band on agarose gel). Resulting PCR products were visualized on a 1.5\% agarose gel. Positive amplicons (visualization of a $300 \mathrm{bp} 16 \mathrm{~S}$ band on the agarose gel) were normalized using the SequalPrep normalization kit (Thermo Fisher Scientific A1051001), and resultant PCR products were sequenced by the McMaster Genomics Facility (Hamilton, ON), using the Illumina MiSeq platform. The resulting 16S sequences were processed as previously described, by sllp, our in-house data pipeline ${ }^{45}$. As per the McMaster Genomics Facility protocol, samples not yielding a PCR product for the $16 \mathrm{~S}$ rRNA gene were not sent for sequencing. These samples were considered to be negative. A representative $1.5 \%$ agarose gel demonstrating the presence or absence of a PCR product following the two-stage PCR for the 16S rRNA gene in our endometrial samples is presented in Supplemental Fig. 1. There were 3 endometrial biopsies (2 Cases, and 1 Control) that did not yield a PCR product for the $16 \mathrm{~S}$ rRNA gene and were thus considered negative (the band at $\sim 80 \mathrm{bp}$ represents dimers of primers + Illumina adapters/barcode/priming region). Therefore, subsequent analyses were performed on the remaining 12 Cases and 9 Controls.

Alpha-diversity excluding singletons was calculated using the sllp pipeline ${ }^{45}$, and QIIME version 1.9.1-dev. Ten rarefaction tables with 3848 sequences were used. Observed species, Chaol, and Shannon Diversity were graphed and analyzed using GraphPad Prism (GraphPad Software Inc., La Jolla, CA). Linear discriminant analysis (LDA) effect size (LEfSe) ${ }^{47}$ (https://huttenhower.sph.harvard.edu/galaxy/) was used to determine if there were significant taxonomic differences in the endometrial microbiota of cases and symptomatic controls. Alpha values of 0.05 , and the 2.0 threshold for logarithmic LDA score for discriminative features were selected for LDA analysis. Taxa bar charts, Bray-Curtis dissimilarity PCoAs, the gap statistic, and heatmaps were generated in R version 3.2.3 (R Core Team, 2015) as described ${ }^{46}$. For species level estimations (described in ${ }^{46}$ ) in the heatmap, most of the OTUs could not be resolved to the species level (100\% identity and coverage on NCBI's nucleotide BLAST: https://blast.ncbi.nlm.nih.gov/Blast.cgi using the 16S rRNA database), and are thus reported as the resultant genera from our $16 \mathrm{~S}$ sequencing.

Statistical analysis. Researchers were blinded to experimental groups until data analysis. Demographic characteristics were tested for normality and groups were statistically compared using SigmaPlot (SigmaPlot 10.0, Systat Software Inc., San Jose, CA, USA). Two-tailed Student's t-tests (for age of the study participants, age at menarche, years since menarche, and duration of menstrual bleeding), Fisher's exact tests (for smoking status), and Chi square (for ethnicity, occupational status, and menstrual cycle stage) were used to compare Cases and Controls. A p value $\leq 0.05$ was considered significant for all statistical tests employed.

The $16 \mathrm{~S}$ data was tested for normality and alpha-diversity metrics were statistically compared using multiple unpaired t-tests, corrected for multiple comparisons using the Holm-Sidak method (GraphPad Software Inc., La Jolla, CA). Beta-diversity was assessed between groups by permutational multivariate analysis of variance (PERMANOVA), using Bray-Curtis dissimilarity distance matrices and employing the adonis function in the vegan package $^{48}$ in $R$. Data are presented as mean \pm SEM, unless otherwise indicated.

\section{Results}

Study participants. The characteristics describing the study participants included in this report are shown in Supplemental Table 1. Mean age, ethnicity, occupational status, smoking status, number of years since menarche, duration of menstrual bleeding, and stage of the menstrual cycle at surgery were similar between symptomatic controls and patients affected by endometriosis. However, symptomatic controls did report a significantly earlier age at menarche $(11.6 \pm 1.0$ vs. $13.0 \pm 1.3$ years, $p=0.015)$.

Endometrial microbiota. Three endometrial biopsies (2 Cases, and 1 Symptomatic Control) did not yield a PCR product for the 16S rRNA gene and were thus considered negative. Therefore, 16S rRNA gene analyses were performed on the remaining 12 Cases and 9 Symptomatic Controls. The minimum number of $16 \mathrm{~S}$ sequences read during sequencing was 867 , and the maximum was 20,113 . The average number of sequence reads for all study participants was $9155.6 \pm 1613.8$.

Diversity of the endometrial microbiota of people with endometriosis is greater than in symptomatic controls. First, we sought to compare bacterial richness and evenness in the endometrial microbiota of patients with surgically confirmed endometriosis $(\mathrm{N}=12)$ versus surgically confirmed symptomatic controls ( $\mathrm{N}=9$; people with pelvic pain, but not endometriosis). Three alpha-diversity (estimates of species diversity within the endometrial microbiota of an individual) metrics were used to compare the endometrial microbiota of these groups (Fig. 1). No significant differences in Observed Species (Fig. 1A) or Chao 1 Richness (Fig. 1B) were observed at the levels of rarefaction where graphs levelled off and the greatest number of samples was retained (multiple unpaired t-tests, corrected for multiple comparisons using the Holm-Sidak method). However, people with endometriosis had significantly greater bacterial diversity as assessed by the Shannon Diversity 
A

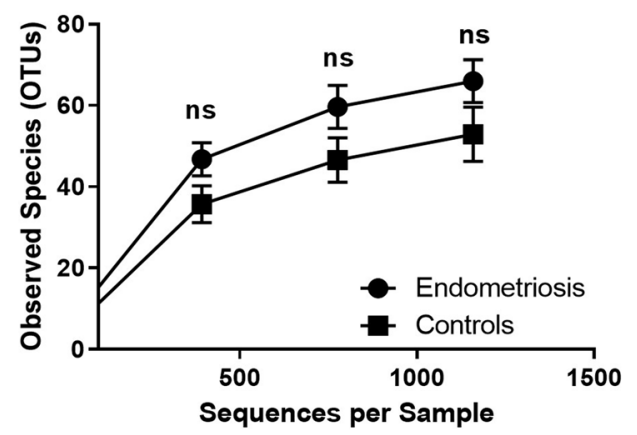

C

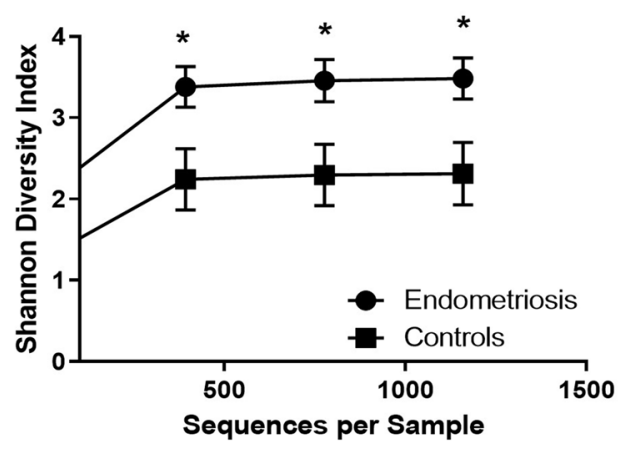

B

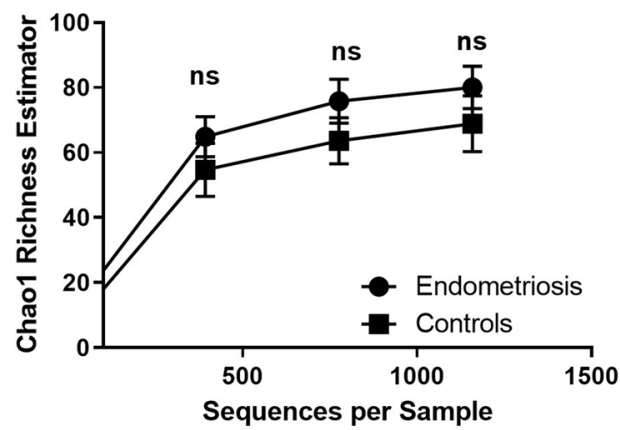

Relative Abundance of Top 10 Taxa in Endometrial Microbiota (\%)

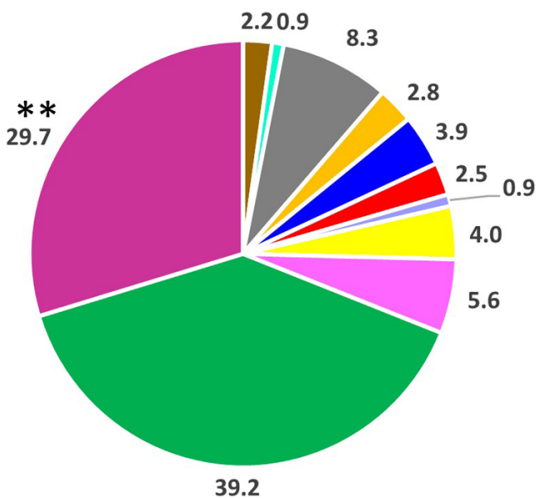

Cases (Endometriosis) $\mathbf{N}=12$

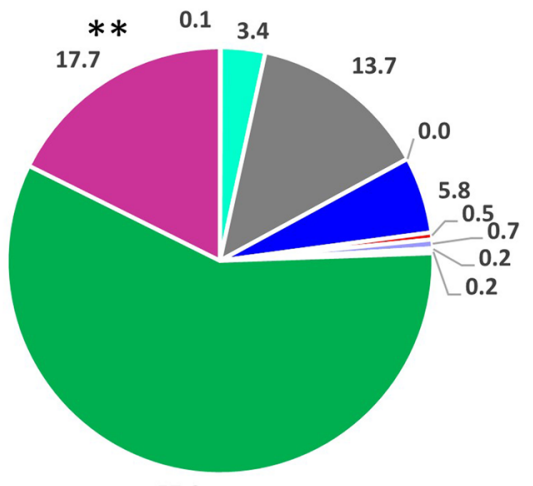

57.9

\section{Legend}

Mycoplasma Lactobacillus Lachnospiraceae (other) Allobaculum Moraxella Bacteroidales Lachnospiraceae Lactococcus Bifidobacterium Escherichia Others

Figure 1. Endometrial microbiota of patients with endometriosis is significantly more diverse than symptomatic controls. Three alpha-diversity metrics were used to compare bacterial richness and evenness within the endometrial microbiota of patients with surgically confirmed endometriosis $(\mathrm{N}=12)$ and surgically confirmed controls (with pelvic pain, but not endometriosis, $N=9$ ). (A) No significant differences in Observed Species or (B) Chao 1 Richness were observed at the levels of rarefaction where graphs levelled off and the greatest number of samples was retained (multiple unpaired t-tests, corrected for multiple comparisons using the Holm-Sidak method). (C) Patients with endometriosis had significantly greater bacterial diversity as assessed by the Shannon Diversity Index at all levels of rarefaction where the graph levelled off and the greatest number of samples was retained (adjusted $p \leq 0.05$; multiple unpaired t-tests, corrected for multiple comparisons using the Holm-Sidak method). (D) The average percent relative abundance of the top 10 taxa in the endometrial microbiota of cases and controls was plotted as a pie chart and the proportion of taxa assigned to the 'Others' category was significantly greater in patients with endometriosis than symptomatic controls (unadjusted $p=0.007$; adjusted $p=0.07$, Mann-Whitney $\mathrm{U}$ test without/with correction for multiple comparisons using the Holm-Sidak method). ${ }^{*} p \leq 0.05,{ }^{* *} p \leq 0.01$. Data is presented as mean \pm SEM $(\mathbf{A}-\mathbf{C})$ and as a percentage (D). Ns: not significant. OTUs: operational taxonomic units. \#\#\#: resolved to bacterial order, \#\#\#: resolved to bacterial family. 
Index at all levels of rarefaction (Fig. 1C) (adjusted $p \leq 0.05$; multiple unpaired t-tests, corrected for multiple comparisons using the Holm-Sidak method). We also plotted the average percent relative abundance of the top 10 taxa in the endometrial microbiota of cases and controls, and found the proportion of taxa assigned to the 'Others' category was significantly greater in patients with endometriosis than symptomatic controls (Fig. 1D) $(29.7 \pm 3.1 \%$ in Cases vs. $17.7 \pm 2.9 \%$ in Controls; unadjusted $p=0.007$, Mann-Whitney U Test). However, after adjusting for multiple comparisons (Holm-Sidak method), the relationship verged on significance (adjusted $p=0.07)$. No significant differences between the other top 10 taxa were observed.

When we repeated these analyses only including people with stage 4 endometriosis $(\mathrm{N}=10)$ versus controls $(\mathrm{N}=9)$ (Supplemental Fig. 2), we saw the same results as above (no significant difference in Observed Species or Chao 1 (multiple unpaired t-tests, corrected for multiple comparisons using the Holm-Sidak method), and a greater significant difference in Shannon Diversity Index at all levels of rarefaction (adjusted $p \leq 0.01$; multiple unpaired t-tests, corrected for multiple comparisons using the Holm-Sidak method)). We also saw the proportion of taxa assigned to the 'Others' category was significantly greater in patients with stage 4 endometriosis than symptomatic controls ( $31.3 \pm 3.5 \%$ in Stage 4 Cases vs. $17.7 \pm 2.9 \%$ in Controls; unadjusted $p=0.008$, Mann-Whitney U Test). However, after adjusting for multiple comparison (Holm-Sidak method), the relationship verged on significance (adjusted $p=0.08$ ).

Beta-diversity of the endometrial microbiota of people with and without endometriosis. Next, we examined beta-diversity (estimates of species diversity within the endometrial microbiota of one group versus another). The top 20 bacterial genera in the endometrial microbiota were plotted by relative abundance as individual taxa bar charts (Fig. 2A) and compared between patients with surgically confirmed endometriosis $(\mathrm{N}=12)$ and surgically confirmed symptomatic controls $(\mathrm{N}=9)$. Each bar represents the endometrial microbiota of one person. Each colour represents a different genus of bacteria, as indicated in the legend. Endometrial microbiota are ordered left to right in descending order of the relative abundance of lactobacilli. Patients with endometriosis had endometrial flora that verged on being significantly different from the endometrial microbiota of surgically confirmed, symptomatic controls ( $\beta$-diversity, $p=0.09$, PERMANOVA). We also plotted a principal coordinate analysis plot (PCoA) to demonstrate the beta-diversity of the endometrial microbiota at the OTU level based on the Bray-Curtis dissimilarity matrix (Fig. 2B). Endometrial microbiota did not appear to cluster by disease status (endometriosis vs. controls) during principal coordinates analysis, however three clusters were identified in the data using the gap statistic (K-means clustering) (Fig. 3A). PCoA ordination and the Bray-Curtis dissimilarity distance were used to construct a heatmap (Fig. 3B).

Taxonomic differences in the endometrial microbiota of cases and symptomatic controls. Finally, we wanted to determine which bacterial taxa were differentially represented (in terms of relative abundance) in the endometrial microbiota of patients with endometriosis versus symptomatic controls. We performed a LefSe analysis that separated the endometrial microbiota of cases from symptomatic controls based on relative abundance of the bacterial genera listed (Fig. 4A). Our LEfSe analysis revealed enrichment of taxa including bacteria in the Actinobacteria phylum, Oxalobacteraceae and Streptococcaceae families, and Tepidimonas genus in patients with endometriosis, while symptomatic controls had enrichment of the Burkholderiaceae family, and Ralstonia genus. A cladogram was created to show the relationship between bacterial taxa and highlight the differential taxa (in terms of relative abundance) in the endometrial microbiota of patients with endometriosis and symptomatic controls (Fig. 4B).

\section{Discussion}

Herein, we compared the endometrial microbiota recovered from endometrial biopsies of patients with surgically confirmed presence or absence of endometriosis (cases versus symptomatic controls-patients with pain but without endometriosis). We demonstrate the presence of an endometrial microbiota in the uterus of patients with endometriosis that is more diverse (greater Shannon Diversity Index (an estimator of species richness and evenness ${ }^{49}$ ), and greater proportion of 'Other' taxa) than that of symptomatic controls (patients with pelvic pain, but surgical absence of endometriosis). This suggests although the total number of bacterial species in the endometrial microbiotas is similar (Observed Species and Chao 1 Richness were comparable between groups), the species evenness differs between Cases and Controls. In other words, there is more variability in species abundance in Cases as compared to Controls ${ }^{49}$. Although the effect of enhanced diversity of the endometrial microbiota in people with endometriosis is presently unknown, perhaps this variability differentially induces an immune response, ultimately contributing to disease pathophysiology. The relative abundance of bacterial taxa enriched in the endometrial microbiota of patients with endometriosis belonged to the Actinobacteria phylum (Gram-positive), Oxalobacteraceae (Gram-negative) and Streptococcaceae (Grampositive) families, and Tepidimonas (Gram-negative) genus, while those enriched in the symptomatic controls belonged to the Burkholderiaceae (Gram-negative) family, and Ralstonia (Gram-negative) genus. Our results align with a recent systematic review ${ }^{50}$ that found 9 of 15 studies profiling the microbiotas (vaginal, cervical, endometrial, peritoneal fluid, endometriotic lesion, and/or gut) identified Gram-negative bacterial taxa that were significantly enriched in people with endometriosis, which may provide additional support for a putative link between bacterial endotoxins/LPS (part of the outer membrane of Gram-negative bacteria) and endometriosis as proposed by the bacterial contamination hypothesis (reviewed in ${ }^{38,39}$ ). Similar to Chen et al., 2017 who used endometrial swabs to profile uterine bacteria by $16 \mathrm{~S}$ rRNA sequencing, none of the endometrial microbiotas profiled in our study were Lactobacillus-dominant (>90\% Lactobacillus species) ${ }^{26}$. This contrasts with several other studies ${ }^{25,27-29,31,33,51}$ where they report endometrial microbiotas that are Lactobacillus-dominant. Instead, the major taxa represented in our study population were Mycoplasma (Gram-negative), 'Others', Lactobacillus 
A

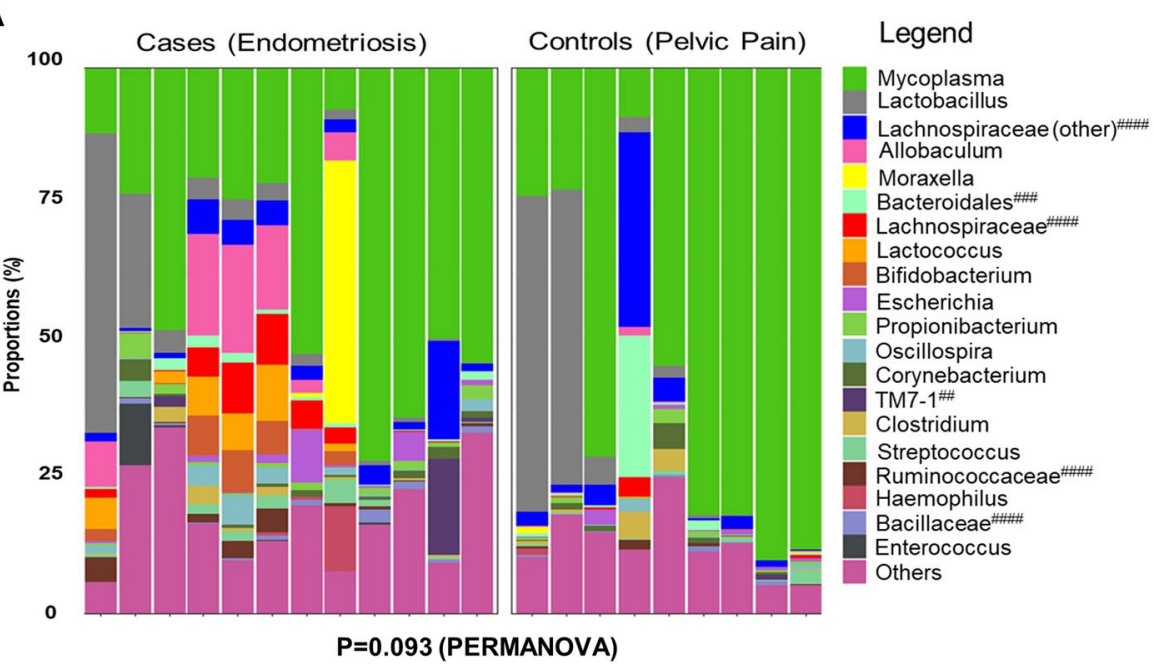

B

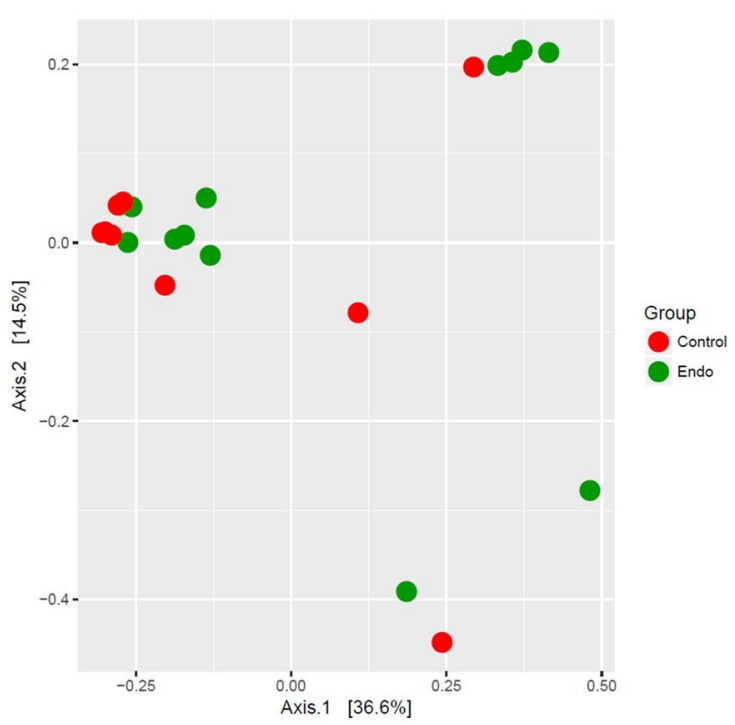

Figure 2. Beta-diversity of patients with endometriosis verges on being significantly greater than symptomatic controls. (A) The top 20 bacterial genera in the endometrial microbiota were plotted by relative abundance as taxa bar charts and compared between patients with surgically confirmed endometriosis $(\mathrm{N}=12)$ and surgically confirmed controls $(\mathrm{N}=9)$. Each bar represents the endometrial microbiota of one individual. Each colour represents a different genus of bacteria, as indicated in the legend. Endometrial microbiota are ordered left to right in descending order of the relative abundance of lactobacilli. Patients with endometriosis (as a group) had an endometrial flora that verged on being significantly different from the endometrial microbiota of surgically confirmed, symptomatic controls ( $\beta$-diversity, $p=0.09$, PERMANOVA). \#\#: Resolved to bacterial class. \#\#\#: Resolved to bacterial order. \#\#\#: Resolved to bacterial family. (B) The principal coordinate analysis plots (PCoA) demonstrated the beta-diversity of the endometrial microbiota at the OTU level based on the BrayCurtis dissimilarity matrix (endometriosis $=$ green dots, controls $=$ red dots). Axes = eigenvalues, a metric whose magnitude indicates the amount of variation captured in the PCoA axis.

(Gram-positive), Lachnospiraceae (other) (Gram-positive), Allobaculum (Gram-positive), Moraxella (Gramnegative), Bacteroidales (Gram-negative), Lachnospiraceae (Gram-positive), Lactococcus (Gram-positive), Bifidobacterim (Gram-positive), and Escherichia (Gram-negative). We suspect this could be a result of using different extraction methods and biological materials for $16 \mathrm{~S}$ rRNA sequencing (endometrial fluid ${ }^{25,29,52}$; embryo transfer catheter tip ${ }^{24,27}$; uterine washings ${ }^{51}$ ) and different populations of patients (fertility patients ${ }^{25,27-29,51}$ ). In fact, it was recently reported that the microbiota recovered from endometrial tissue samples was not fully reflected in paired endometrial fluid ${ }^{53}$, supporting the notion that different biological materials from the same anatomical location can yield different microbiota compositions. Furthermore, we did not find differences in demographics between our cases and controls, except that symptomatic controls had a significantly earlier age at menarche. It is also important to note that three endometrial biopsies (2 Cases, and 1 Symptomatic Control) did not yield 
A

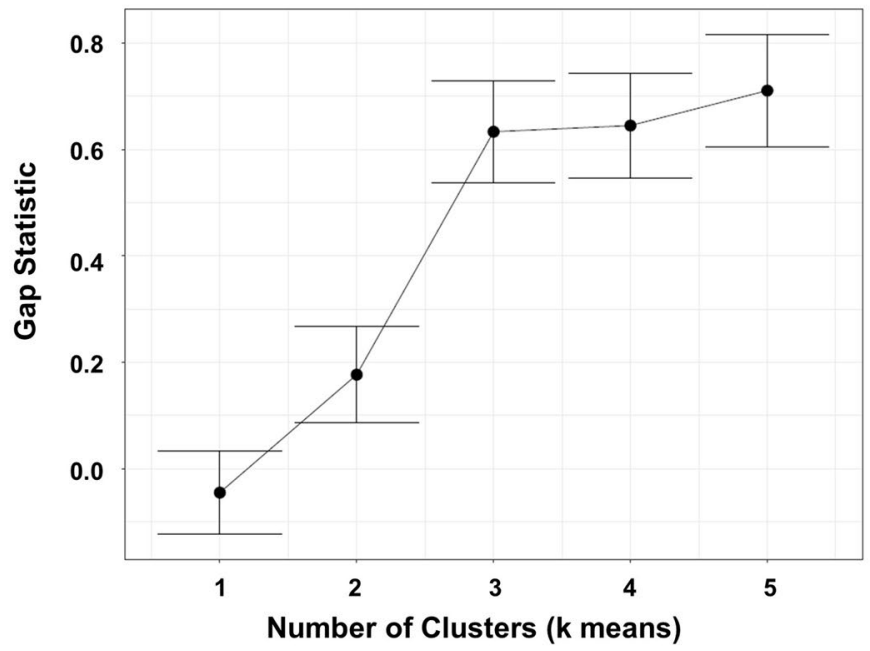

B
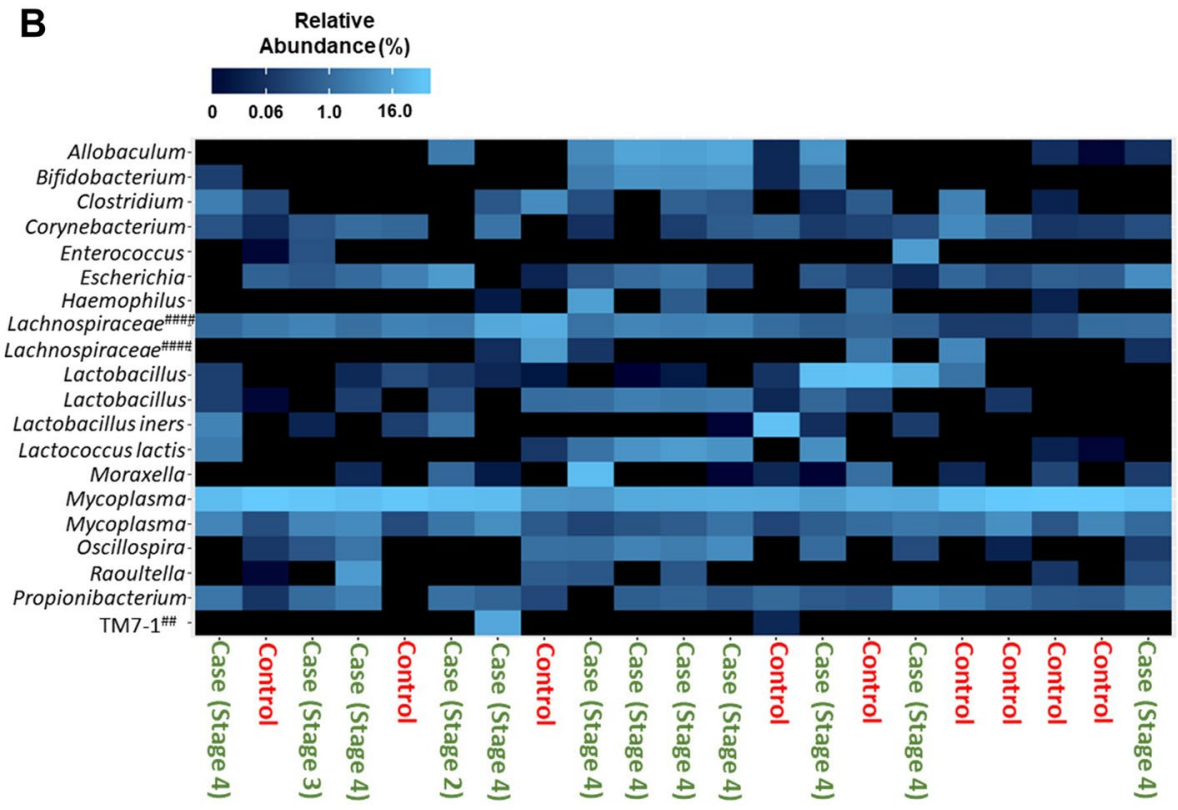

Figure 3. Examination of endometrial microbiota clustering from patients with endometriosis and symptomatic controls. (A) The gap statistic was calculated to give an estimation of the number of clusters found in the PCoA. Three clusters were present in the data, as indicated by the plateau in the gap statistic, which occurred at a value of 3 in this graph. (B) A heatmap of the top 20 bacterial taxa based on Bray-Curtis dissimilarity distance and PCoA ordination demonstrated the endometrial microbiota (columns) by disease status (Endometriosis Cases vs. Symptomatic Controls). Taxa are ordered alphabetically along the y-axis. \#\# Resolved to bacterial class. \#\#\#: Resolved to bacterial family.

a PCR product for the $16 \mathrm{~S}$ rRNA gene, and were thus considered negative. This may suggest that not everyone harbours an endometrial microbiota.

The bacteria in the human microbiotas co-evolved with their hosts. Much of our knowledge of bacterial-host interactions has been gleaned from the high diversity gut microbiota, which is critical in modulating host immunity (reviewed in ${ }^{54}$ ). However, the female reproductive tract microbiotas (vaginal, cervical, endometrial) are lower in diversity ${ }^{26}$ and distinct from the gut ${ }^{55}$. Although the role of the endometrial microbiota in human health and disease is largely unknown, it is becoming increasingly clear that perturbations in endometrial bacteria are associated with pathologies like endometriosis ${ }^{26,33,51,56}$, endometrial $\operatorname{cancer}^{35,36}$, uterine fibroids ${ }^{30}$, and in success or failure of pregnancy following IVF $^{21-23,25}$. Further, perturbations in bacterial populations are not limited to the endometrium of people with endometriosis, but may be more widespread (reviewed in $^{40}$ ). Differences in the vaginal ${ }^{26,57}$, cervical ${ }^{26,51,57-59}$, and gut microbiotas ${ }^{60,61}$ are reported between people with endometriosis and controls in some studies, but not all ${ }^{62}$. Similar to our observations in the endometrial microbiota, two 
A
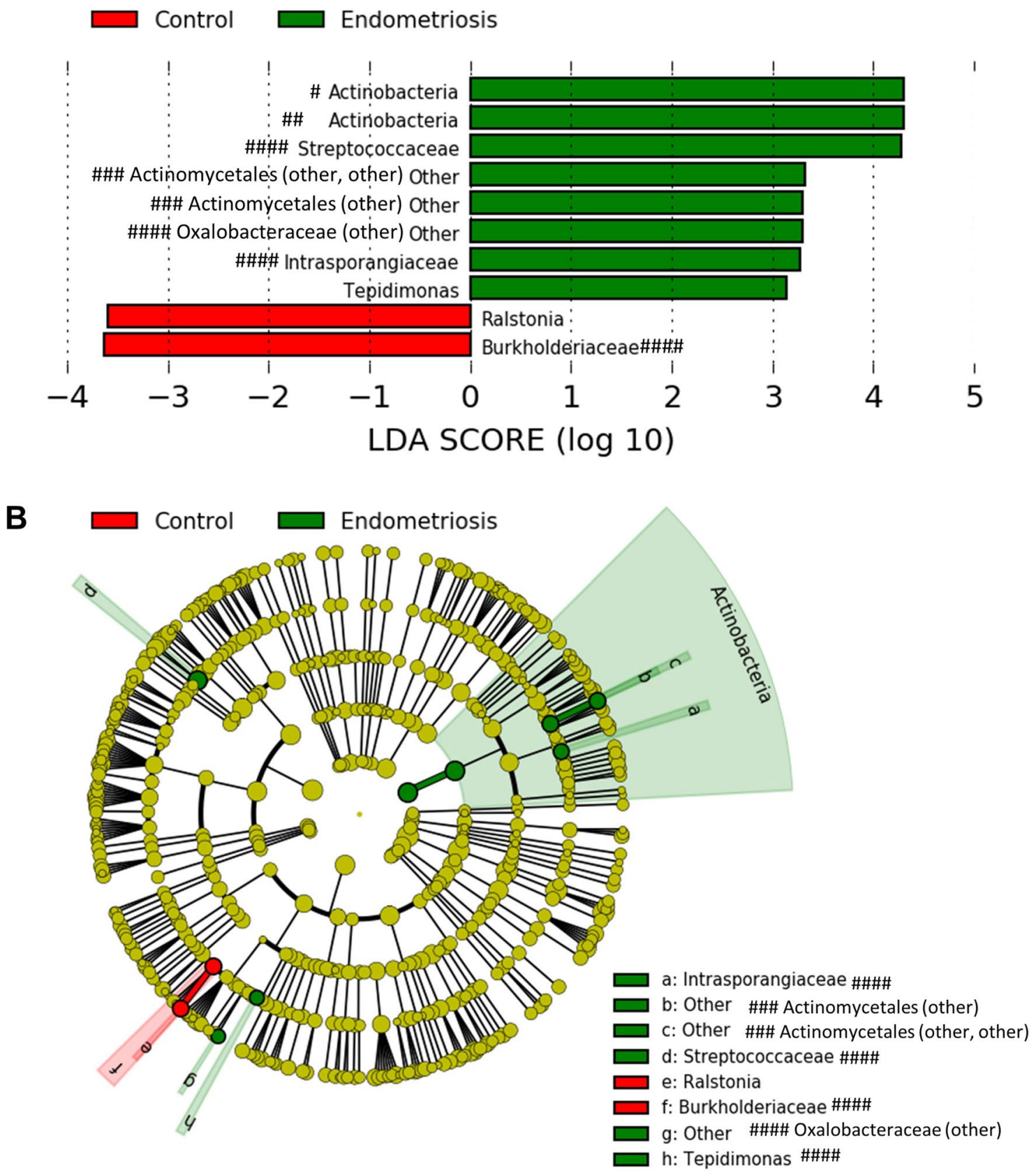

Figure 4. Differential bacterial taxa in the endometrial microbiota of patients with endometriosis versus symptomatic controls. (A) Linear Discriminant Analysis effect size (LefSe) analysis separated the endometrial microbiota of patients with endometriosis from control women with pelvic pain based on relative abundance of the bacterial genera listed, using 2.0 as a threshold for discriminative features, and $p \leq 0.05$ for statistical tests. LEfSe analysis revealed enrichment of taxa including bacteria in the Actinobacteria phylum, Oxalobacteraceae and Streptococcaceae families, and Tepidimonas genus in patients with endometriosis, while symptomatic controls were found to have enrichment of the Burkholderiaceae family, and Ralstonia genus. (B) Cladogram of differential taxa in the endometrial microbiota of patients with endometriosis (in terms of relative abundanceenriched taxa in green) and controls with pelvic pain, but without endometriosis (enriched taxa in red). \#: Resolved to bacterial phylum. \#\#: Resolved to bacterial class. \#\#: Resolved to bacterial order. \#\#\#: Resolved to bacterial family.

independent reports found increased diversity in the cervical microbiota of people with endometriosis compared to controls $s^{51,59}$, further supporting a link between bacterial diversity in the reproductive microbiotas and 
endometriosis. Interestingly, the relationship between bacterial diversity and endometriosis is opposite in the gut, where individuals with endometriosis had lower bacterial diversity than controls ${ }^{60,61}$. Typically reproductive 'health' tends to be associated with a low diversity, Lactobacillus dominant vaginal microbiota (reviewed in ${ }^{63}$ ), while gut 'health' tends to be associated with a diverse microbiota (reviewed in ${ }^{64}$ ). Gut microbiota perturbations have been postulated to contribute to pathogenesis of endometriosis via the regulation of inflammatory processes and estrogen metabolism (reviewed in ${ }^{65}$ ), and gut permeability is increased in patients with endometriosis ${ }^{66}$ which may contribute to the systemic nature of this condition (reviewed in ${ }^{67}$ ). Taken together, our observations in the endometrial microbiota, and those of others in the cervix ${ }^{51,59}$ and gut ${ }^{60}$ suggest that people with endometriosis experience dysbiosis, an imbalance of bacteria, at various sites.

Adding to the evidence that microbiota perturbations are linked with endometriosis are several experimental animal studies demonstrating that lesion development ${ }^{68}$, and presence of disease $\mathrm{e}^{69-71}$ are associated with changes in the gut microbiota of rhesus monkeys and mice. Similar to what Svensson et al., 2021 and Shan et al., 2021 observed in people with endometriosis, $\mathrm{Ni}$ et al., 2020 report that mice with experimentally induced endometriosis had lower bacterial diversity and abundance in the gut microbiota than controls ${ }^{71}$. Another piece of evidence of a link between microbiota perturbations and endometriosis is that certain medications (treatment with letrozole, the Traditional Chinese Medicine Shaofu Zhuyu decoction ${ }^{72}$, or antibiotics ${ }^{73}$ ) reduced endometriotic implant volumes in rats and mice, and was thought to be due in part to restoration of the gut microbiota. However, the challenge in comparing results of microbiota studies in humans and animals is that each study employs different sampling methods and anatomical locations (ie. lower, vs. mid-, vs. upper vagina), sampling timepoints, experimental animals and endometriosis models, 16S rRNA gene sequencing methodologies, and methods of data analysis. Further, from the current literature, it remains unclear which direction the association between bacteria and endometriosis goes; dysbiosis leading to endometriosis, or endometriosis leading to dysbiosis. Nevertheless, increasing evidence suggests perturbations in the microbiotas are associated with endometriosis.

Following reports of bacterial endotoxin in the pelvic cavity and menstrual blood ${ }^{37}$, and "sub-clinical uterine infections" found in patients with endometriosis ${ }^{56}$, Khan et al., 2017 proposed that microbes might activate inflammatory cascades by binding Toll-like receptors, and contribute to endometriotic lesion establishment, growth and progression. This has become known as the 'bacterial contamination hypothesis ${ }^{39}$. In addition to the aforementioned microbiota studies, epidemiological studies also support this hypothesis. A large populationbased study found females with a history of lower genital tract infections were at a 2.01 times higher risk of endometriosis than those without this history ${ }^{74}$, while another population-based retrospective cohort found a 3.02 times greater risk of being diagnosed with endometriosis in females who had previously had pelvic inflammatory disease (pathogenic bacteria spreading from vagina to upper genital tract) ${ }^{75}$. Furthermore, the association between upper genital tract and peritoneal infections and endometriosis was the focus of a recent systematic review ${ }^{76}$. Although the majority of studies on the microbiotas of people with endometriosis focus on the vaginal, cervical, uterine, or gut microbiotas, studies have profiled the bacteria found in endometriotic lesions ${ }^{31,77}$ and extracellular vesicles (ECVs) isolated from peritoneal fluid of patients with endometriomas ${ }^{78}$ by $16 \mathrm{~S}$ sequencing, demonstrating that bacteria can be found in disease lesions and ECVs. Hernandes et al., 2020 compared bacteria recovered from the vaginal fluid, eutopic, and ectopic tissues, and reported similar bacterial profiles (Lactobacillus, Gardnerella, Streptococcus and Prevotella) at these sites. They did note that deep lesions had altered bacterial profiles (less Lactobacillus, more Alishewanella, Enterococcus and Pseudomonas), suggesting that different lesions may support different bacterial populations ${ }^{31}$. Khan et al., 2016 demonstrated that although bacteria could not be cultured from endometriomas (or other non-endometrioma cysts in controls) there was significantly more Streptococcaceae and Staphylococaceae and less Lactobacillacae in endometriomas as compared to non-endometrioma cysts from controls using $16 \mathrm{~S}$ rRNA sequencing ${ }^{77}$. Although some differences in bacteria found in endometriotic lesions have been reported, pathogenic viruses do not follow the same trend $^{79}$. Vestergaard et al., 2010 quantified 11 common pathogenic DNA viruses in the eutopic endometrium of endometriosis cases and controls, and ectopic lesions, revealing low viral prevalence, no significant differences between cases and controls, and no viruses recovered from ectopic lesions. Combined, these epidemiological studies, and microbiota studies profiling bacteria in the endometriotic lesions support the 'bacterial contamination hypothesis', and a role for bacteria in the pathogenesis and/or pathophysiology of endometriosis.

Our study has several strengths including the blinding of researchers to experimental groups until data analysis, the use of $16 \mathrm{~S}$ rRNA gene sequencing to profile the endometrial microbiota of patients with and without endometriosis, the exclusion of people who were using hormone therapies in the 3 months preceding study enrollment, the inclusion of no template negative controls in each PCR run, and the inclusion of patients with surgically confirmed presence or absence of disease. The limitations of our study include our small sample size, lack of information on antibiotic or probiotic use in the months preceding surgery in our population, inability to explore the function of the endometrial bacteria we recovered, and lack of healthy controls (inclusion of which may have helped determine if there is an endometrial microbiota in "healthy" women). Furthermore, our study did not examine other microbiotas of the uterine ecosystem (e.g. virome, mycome, etc.). Additionally, subsequent studies should include additional negative controls for microbiota library preparation (e.g. extraction controls, procedural swabs, hospital room air swabs, and/or other anatomic locations), and positive controls (e.g. mock bacterial communities). Larger, well-controlled studies aimed at understanding the role of bacteria in the pathogenesis and pathophysiology of endometriosis may offer novel insights into new therapeutics for this chronic condition.

In summary, we report that the endometrial microbiota in the uterus of people with endometriosis is more diverse than that of symptomatic controls (with pelvic pain, but surgical absence of endometriosis). The relative abundance of bacterial taxa enriched in the endometrial microbiota of patients with endometriosis belonged to the Actinobacteria phylum, Oxalobacteraceae and Streptococcaceae families, and Tepidimonas genus, while those enriched in the symptomatic controls belonged to the Burkholderiaceae family, and Ralstonia genus. Taken 
together, our study adds to the literature describing perturbations in the endometrial, vaginal, cervical, and gut microbiotas of people with endometriosis.

\section{Data availability}

Raw sequence reads for data included in this manuscript have been deposited in NCBI's Gene Expression Omnibus (GEO) under Accession Number GSE172172.

Received: 1 June 2021; Accepted: 2 September 2021

Published online: 23 September 2021

\section{References}

1. Masciullo, L. et al. A deep insight into pelvic pain and endometriosis: A review of the literature from pathophysiology to clinical expressions. Minerva Obstet. Gynecol. https://doi.org/10.23736/S2724-606X.21.04779-1 (2021).

2. Sampson, J. A. Peritoneal endometriosis due to menstrual dissemination of endometrial tissue into the peritoneal cavity. Am. J. Obstet. Gynecol. 14, 422-469 (1927).

3. Sourial, S., Tempest, N. \& Hapangama, D. K. Theories on the pathogenesis of endometriosis. Int. J. Reprod. Med. 2014, 1-10 (2014).

4. Ahn, S. H., Singh, V. \& Tayade, C. Biomarkers in endometriosis: Challenges and opportunities. Fertil. Steril. 107, 523-532 (2017).

5. Signorile, P. G. \& Baldi, A. Endometriosis: New concepts in the pathogenesis. Int. J. Biochem. Cell Biol. 42, 778-780 (2010).

6. Signorile, P. G. et al. Embryologic origin of endometriosis: Analysis of 101 human female fetuses. J. Cell Physiol. 227, 1653-1656 (2012).

7. Makiyan, Z. New theory of uterovaginal embryogenesis. Organogenesis 12, 33-41 (2016).

8. Sasson, I. E. \& Taylor, H. S. Stem cells and the pathogenesis of endometriosis. Ann. N. Y. Acad. Sci. 1127, 106-115 (2008).

9. Saunders, P. T. K. \& Horne, A. W. Endometriosis: Etiology, pathobiology, and therapeutic prospects. Cell 184, 2807-2824 (2021).

10. Zondervan, K. T., Becker, C. M. \& Missmer, S. A. Endometriosis. N. Engl. J. Med. 382, 1244-1256 (2020).

11. Peterson, C. M. et al. Risk factors associated with endometriosis: Importance of study population for characterizing disease in the ENDO Study. Am. J. Obstet. Gynecol. 208(451), e1-e11 (2013).

12. Vercellini, P. et al. 'Behind blue eyes' $\dagger$ : The association between eye colour and deep infiltrating endometriosis. Hum. Reprod. 29, 2171-2175 (2014).

13. Shafrir, A. L. et al. Risk for and consequences of endometriosis: A critical epidemiologic review. Best Pract. Res. Clin. Obstet. Gynaecol. 51, 1-15 (2018).

14. Sirohi, D., Al Ramadhani, R. \& Knibbs, L. D. Environmental exposures to endocrine disrupting chemicals (EDCs) and their role in endometriosis: A systematic literature review. Rev. Environ Health 36, 101-115 (2020).

15. Halme, J., Hammond, M. G., Hulka, J. F., Raj, S. G. \& Talbert, L. M. Retrograde menstruation in healthy women and in patients with endometriosis. Obstet. Gynecol. 64, 151-154 (1984).

16. Murgia, F. et al. Metabolic profile of patients with severe endometriosis: A prospective experimental study. Reprod. Sci. 28, 728-735 (2021).

17. Koninckx, P. R. et al. Pathogenesis of endometriosis: The genetic/epigenetic theory. Fertil. Steril. 111, 327-340 (2019).

18. Angioni, S. et al. Genetic characterization of endometriosis patients: Review of the literature and a prospective cohort study on a Mediterranean population. Int. J. Mol. Sci. 21, 1-24 (2020).

19. Peterson, J. et al. The NIH human microbiome project. Genome Res. 19, 2317-2323 (2009).

20. Sender, R., Fuchs, S. \& Milo, R. Revised estimates for the number of human and bacteria cells in the body. PLoS Biol. 14, 1-14 (2016).

21. Egbase, P. E. et al. Incidence of microbial growth from the tip of the embryo transfer catheter after embryo transfer in relation to clinical pregnancy rate following in-vitro fertilization and embryo transfer. Hum. Reprod. (Oxford, England) 11, 1687-1689 (1996).

22. Moore, D. E. et al. Bacteria in the transfer catheter tip influence the live-birth rate after in vitro fertilization. Fertil. Steril. 74, 1118-1124 (2000).

23. Selman, H. et al. Examination of bacterial contamination at the time of embryo transfer, and its impact on the IVF/pregnancy outcome. J. Assist. Reprod. Genet. 24, 395-399 (2007).

24. Franasiak, J. M. et al. Endometrial microbiome at the time of embryo transfer: Next-generation sequencing of the $16 \mathrm{~S}$ ribosomal subunit. J. Assist. Reprod. Genet. 33, 129-136 (2016).

25. Moreno, I. et al. Evidence that the endometrial microbiota has an effect on implantation success or failure. Am. J. Obstet. Gynecol. 215, 684-703 (2016).

26. Chen, C. et al. The microbiota continuum along the female reproductive tract and its relation to uterine-related diseases. Nat. Commun. 8, 1-11 (2017)

27. Tao, X. et al. Characterizing the endometrial microbiome by analyzing the ultra-low bacteria from embryo transfer catheter tips in IVF cycles: Next generation sequencing (NGS) analysis of the 16 S ribosomal gene. Hum. Microbiome J. 3, 15-21 (2017).

28. Kyono, K., Hashimoto, T., Nagai, Y. \& Sakuraba, Y. Analysis of endometrial microbiota by $16 S$ ribosomal RNA gene sequencing among infertile patients: A single-center pilot study. Reprod. Med. Biol. 17, 297-306 (2018).

29. Kitaya, K., Nagai, Y., Arai, W., Sakuraba, Y. \& Ishikawa, T. Characterization of microbiota in endometrial fluid and vaginal secretions in infertile women with repeated implantation failure. Mediators Inflamm. 2019, 1-10 (2019).

30. Winters, A. D. et al. Does the endometrial cavity have a molecular microbial signature?. Sci. Rep. 9, 1-17 (2019).

31. Hernandes, C. et al. Microbiome profile of deep endometriosis patients: Comparison of vaginal fluid, endometrium and lesion. Diagnostics 10, 1-11 (2020).

32. Moreno, I. et al. The first glimpse of the endometrial microbiota in early pregnancy. Am. J. Obstet. Gynecol. 222, 296-305 (2020).

33. Wei, W., Zhang, X., Tang, H., Zeng, L. \& Wu, R. Microbiota composition and distribution along the female reproductive tract of women with endometriosis. Ann. Clin. Microbiol. Antimicrob. 19, 1-8 (2020).

34. Baker, J. M., Chase, D. M. \& Herbst-Kralovetz, M. M. Uterine microbiota: Residents, tourists, or invaders?. Front. Immunol. 9, $1-16$ (2018).

35. Walther-António, M. R. S. et al. Potential contribution of the uterine microbiome in the development of endometrial cancer. Genome Med. 8, 1-15 (2016).

36. Lu, W. et al. Dysbiosis of the endometrial microbiota and its association with inflammatory cytokines in endometrial cancer. Int. J. Cancer 148, 1708-1716 (2021).

37. Khan, K. N. et al. Escherichia coli contamination of menstrual blood and effect of bacterial endotoxin on endometriosis. Fertil. Steril. 94, 2860-3.e1-3 (2010).

38. Puca, J. \& Hoyne, G. F. Microbial dysbiosis and disease pathogenesis of endometriosis, could there be a link?. Allied J. Med. Res. 1(1), 1-9 (2016).

39. Khan, K. N. et al. Bacterial contamination hypothesis: A new concept in endometriosis. Reprod. Med. Biol. 17, 125-133 (2018). 
40. Leonardi, M., Hicks, C., El-Assaad, F., El-Omar, E. \& Condous, G. Endometriosis and the microbiome: A systematic review. BJOG 127, 239-249 (2020).

41. Wessels, J. M. et al. Medroxyprogesterone acetate alters the vaginal microbiota and microenvironment in women and increases susceptibility to HIV-1 in humanized mice. Dis. Model. Mech. 12, 1-13 (2019).

42. ASRM. Revised American Society for Reproductive Medicine classification of endometriosis: 1996. Fertil. Steril. 67, 817-821 (1997).

43. Noyes, R. W., Hertig, A. T. \& Rock, J. Dating the endometrial biopsy. Fertil. Steril. 1, 3-11 (1950).

44. Bartram, A. K., Lynch, M. D. J., Stearns, J. C., Moreno-Hagelsieb, G. \& Neufeld, J. D. Generation of multimillion-sequence 16S rRNA gene libraries from complex microbial communities by assembling paired-end illumina reads. Appl. Environ. Microbiol. 77, 3846-3852 (2011).

45. Whelan, F. J. \& Surette, M. G. A comprehensive evaluation of the sllp pipeline for $16 \mathrm{~S}$ rRNA gene sequencing analysis. Microbiome 5, 1-13 (2017).

46. Wessels, J. M. et al. Association of high-risk sexual behaviour with diversity of the vaginal microbiota and abundance of Lactobacillus. PLoS ONE 12, e0187612 (2017).

47. Segata, N. et al. Metagenomic biomarker discovery and explanation. Genome Biol. 12, R60 (2011).

48. Oksanen, J. B., Guillaume, F., Friendly, M., Kindt, R., Legendre, P., McGlinn, D., Minchin, P. R., O'Hara, R. B., Simpson, G. L., Solymos, P. M., Stevens, H. H., Szoecs, E. Wagner, H. vegan: Community ecology package. In R package version 24-1 (2016).

49. Kim, B. R. et al. Deciphering diversity indices for a better understanding of microbial communities. J. Microbiol. Biotechnol. 27, 2089-2093 (2017).

50. D’Alterio, M. N. et al. Possible role of microbiome in the pathogenesis of endometriosis. Minerva Obstet. Gynecol. 73, 193-214 (2021).

51. Cregger, M. A. et al. Reproductive microbiomes: Using the microbiome as a novel diagnostic tool for endometriosis. Reprod. Immunol. Open Access 02, 1-7 (2017).

52. Kyono, K., Hashimoto, T., Kikuchi, S., Nagai, Y. \& Sakuraba, Y. A pilot study and case reports on endometrial microbiota and pregnancy outcome: An analysis using $16 \mathrm{~S}$ rRNA gene sequencing among IVF patients, and trial therapeutic intervention for dysbiotic endometrium. Reprod. Med. Biol. 18, 72-82 (2019).

53. Liu, Y. et al. Systematic comparison of bacterial colonization of endometrial tissue and fluid samples in recurrent miscarriage patients: Implications for future endometrial microbiome studies. Clin. Chem. 64, 1743-1752 (2018).

54. Belkaid, Y. \& Hand, T. W. Role of the microbiota in immunity and inflammation. Cell 157, 121-141 (2014).

55. Aagaard, K. et al. The placenta harbors a unique microbiome. Sci. Transl. Med. 6, 1-12 (2014).

56. Khan, K. N. et al. Intra-uterine microbial colonization and occurrence of endometritis in women with endometriosis†. Hum. Reprod. 29, 2446-2456 (2014).

57. Ata, B. et al. The endobiota study: comparison of vaginal, cervical and gut microbiota between women with stage $3 / 4$ endometriosis and healthy controls. Sci. Rep. 9, 1-9 (2019).

58. Chen, S. et al. Microbiome of the lower genital tract in Chinese women with endometriosis by 16s-rRNA sequencing technique: A pilot study. Ann. Transl. Med. 8, 1-13 (2020).

59. Akiyama, K. et al. Molecular detection of microbial colonization in cervical mucus of women with and without endometriosis. Am. J. Reprod. Immunol. 82, 1-9 (2019).

60. Svensson, A., Brunkwall, L., Roth, B., Orho-Melander, M. \& Ohlsson, B. Associations between endometriosis and gut microbiota. Reprod. Sci. 28, 2367-2377 (2021).

61. Shan, J., Ni, Z., Cheng, W., Zhou, L., Zhai, D., Sun, S. \& Yu, C. Gut microbiota imbalance and its correlations with hormone and inflammatory factors in patients with stage 3/4 endometriosis. Arch. Gynecol. Obstet. https://doi.org/10.1007/s00404-021-06057-z (2021).

62. Perrotta, A. R. et al. The vaginal microbiome as a tool to predict rASRM stage of disease in endometriosis: A pilot study. Reprod. Sci. 27, 1064-1073 (2020).

63. Moosa, Y., Kwon, D., de Oliveira, T. \& Wong, E. B. Determinants of vaginal microbiota composition. Front. Cell Infect. Microbiol. 10, $1-9(2020)$

64. Lozupone, C. A., Stombaugh, J. I., Gordon, J. I., Jansson, J. K. \& Knight, R. Diversity, stability and resilience of the human gut microbiota. Nature 489, 220-230 (2012).

65. Laschke, M. W. \& Menger, M. D. The gut microbiota: A puppet master in the pathogenesis of endometriosis?. Am. J. Obstet. Gynecol. 215(68), e1-e4 (2016).

66. Viganó, D. et al. How is small bowel permeability in endometriosis patients? A case control pilot study. Gynecol. Endocrinol. 36, 1010-1014 (2020).

67. Taylor, H. S., Kotlyar, A. M. \& Flores, V. A. Endometriosis is a chronic systemic disease: Clinical challenges and novel innovations. Lancet 397, 839-852 (2021).

68. Hantschel, J. et al. Effect of endometriosis on the fecal bacteriota composition of mice during the acute phase of lesion formation. PLoS ONE 14, 1-12 (2019).

69. Bailey, M. T. \& Coe, C. L. Endometriosis is associated with an altered profile of intestinal microflora in female rhesus monkeys. Hum. Reprod. 17, 1704-1708 (2002).

70. Yuan, M. et al. Endometriosis induces gut microbiota alterations in mice. Hum. Reprod. 33, 607-616 (2018).

71. Ni, Z. et al. Correlation of fecal metabolomics and gut microbiota in mice with endometriosis. Am. J. Reprod. Immunol. 84, 1-14 (2020).

72. Cao, Y., Jiang, C., Jia, Y., Xu, D. \& Yu, Y. Letrozole and the traditional Chinese medicine, Shaofu Zhuyu decoction, reduce endometriotic disease progression in rats: A potential role for gut microbiota. Evid. Based Complement Alternat. Med. 2020, 1-14 (2020).

73. Chadchan, S. B. et al. Antibiotic therapy with metronidazole reduces endometriosis disease progression in mice: A potential role for gut microbiota. Hum. Reprod. 34, 1106-1116 (2019).

74. Lin, W. C., Chang, C. Y. Y., Hsu, Y. A., Chiang, J. H. \& Wan, L. Increased risk of endometriosis in patients with lower genital tract infection: A nationwide cohort study. Medicine (United States) 95, 1-8 (2016).

75. Tai, F. W., Chang, C. Y. Y., Chiang, J. H., Lin, W. C. \& Wan, L. Association of pelvic inflammatory disease with risk of endometriosis: A nationwide cohort study involving 141,460 individuals. J. Clin. Med. 7, 1-11 (2018).

76. Koninckx, P. R. et al. Infection as a potential cofactor in the genetic-epigenetic pathophysiology of endometriosis: A systematic review. Facts Views Vis. Obgyn. 11, 209-216 (2019).

77. Khan, K. N. et al. Molecular detection of intrauterine microbial colonization in women with endometriosis. Eur. J. Obstet. Gynecol. Reprod. Biol. 199, 69-75 (2016).

78. Lee, S.-R. et al. Altered composition of microbiota in women with ovarian endometrioma: Microbiome analyses of extracellular vesicles in the peritoneal fluid. Int. J. Mol. Sci. 22, 1-12 (2021).

79. Vestergaard, A. L. et al. Low prevalence of DNA viruses in the human endometrium and endometriosis. Arch. Virol. 155, 695-703 (2010). 


\section{Author contributions}

J.M.W., M.A.D., S.K.A. and W.G.F. conceived and designed the experiments; M.A.D. and J.M.W. conducted the experiments and analyzed the results; N.A.L. provided surgical samples; J.M.W. and M.A.D. wrote the manuscript; W.G.F. and N.A.L. supervised the study. W.G.F. acquired funding. J.M.W., M.A.D., S.K.A., N.A.L., and W.G.F. reviewed, edited, and approved the manuscript.

\section{Funding}

This research was supported by an Operating Grant (MOP142230) from the Canadian Institutes of Health Research (WGF), and salary support was provided by the Canadian Institutes of Health Research Fellowship Award (MFE-381806) (JMW). The sponsors did not have any role in study design, collection/analysis/interpretation of data, writing the manuscript, or the decision to submit the article for publication.

\section{Competing interests}

The authors declare no competing interests.

\section{Additional information}

Supplementary Information The online version contains supplementary material available at https:/doi.org/ 10.1038/s41598-021-98380-3.

Correspondence and requests for materials should be addressed to W.G.F.

Reprints and permissions information is available at www.nature.com/reprints.

Publisher's note Springer Nature remains neutral with regard to jurisdictional claims in published maps and institutional affiliations.

(c) (i) Open Access This article is licensed under a Creative Commons Attribution 4.0 International License, which permits use, sharing, adaptation, distribution and reproduction in any medium or format, as long as you give appropriate credit to the original author(s) and the source, provide a link to the Creative Commons licence, and indicate if changes were made. The images or other third party material in this article are included in the article's Creative Commons licence, unless indicated otherwise in a credit line to the material. If material is not included in the article's Creative Commons licence and your intended use is not permitted by statutory regulation or exceeds the permitted use, you will need to obtain permission directly from the copyright holder. To view a copy of this licence, visit http://creativecommons.org/licenses/by/4.0/.

(C) The Author(s) 2021 